\title{
Severe asthma with blood hypereosinophilia associated with JAK2 V617F mutation: a case series
}

\author{
To the Editor:
}

In a large subset of patients with asthma, blood eosinophilia is common, as a marker of T-helper type 2 cell (Th2) inflammation. Hypereosinophilia (HE), defined by blood eosinophil count $>1500$ per $\mathrm{mm}^{3}$, is rare, observed in $0.3 \%$ of asthmatic patients [1]. Classically, in the context of uncontrolled asthma and HE, allergic bronchopulmonary aspergillosis, vasculitis or parasitic infection must be investigated [2]. Asthma is also a feature of HE related to various haematological disorders, although respiratory manifestations are rarely isolated in this setting [2].

Between 2009 and 2018, among 1200 patients evaluated in our reference centre for severe asthma in Bichat Hospital, 34 (3\%) had blood eosinophil count $>1500$ per $\mathrm{mm}^{3}$ on at least two tests. All patients with recurrent $\mathrm{HE}$ were asked to undergo a diagnostic work-up including vitamin B12 and tryptase dosage, blood smear examination and screening for the fusion genes FIPL1-PDGFRA (FIP1-like 1-platelet-derived growth factor receptor- $\alpha$ ) and ETV6-PDGFRB (ETS variant 6-platelet-derived growth factor receptor- $\beta$ ), Janus kinase 2 (JAK2) point mutation, and blood T-cell clone [3, 4]. Bone-marrow analysis was performed when features of lympho- or myeloproliferative disorders were found.

We identified three patients (two females), aged 38 to 65 years, with a JAK2 (V617F) mutation, approximately $9 \%$ of the 34 patients with severe asthma and HE. One patient also had a T-cell clone (patient 2). All had early-onset atopic asthma, with loss of control during adulthood (table 1). HE appeared at age 17, 45 and 55 years, with maximum blood eosinophil count 5540, 4510 and 2000 per $\mathrm{mm}^{3}$, respectively. For all, previous chest computed tomography imaging had revealed transient pulmonary infiltrates, with evidence of eosinophilic alveolitis on bronchoalveolar lavage in two patients. Two patients had a history of venous or arterial thrombosis requiring long-term anticoagulation. A systematic search for vasculitis or aspergillus sensitisation was negative in all patients. In one patient (patient 1), the platelet count was above normal at the time of evaluation, and bone marrow biopsy confirmed a myeloproliferative disorder, which was treated with hydroxyurea. Patient 2 showed high circulating level of B12 vitamin, but normal level of tryptase and bone marrow examination was normal. Patient 3 had no features of a myeloid neoplasm but had venous thromboembolism a few months before the JAK2 mutation finding. For all patients, loss of asthma control appeared less than 1 year before the diagnosis of the JAK2 point mutation. Patients 2 and 3, routinely followed for at least 3 years, currently require low dose prednisone for control of asthma, with normal eosinophil and platelet counts.

JAK2 is a receptor-associated tyrosine kinase activated by several cytokines and growth factors [4]. In eosinophils, JAK2 is phosphorylated and activated after stimulation of the interleukin-5 receptor by the ligand and plays a major role in regulating eosinophilic development [4], migration and activation [5]. It is also involved in inhibiting apoptosis induced by granulocyte-macrophage colony-stimulating factor. A JAK2 gene polymorphism was found associated with increased frequency of virus-induced asthma exacerbations and increased susceptibility to allergic sensitisation to environmental antigens [6]. In murine asthma models, JAK inhibitors inhibit Th2 differentiation, reduce allergen-induced airway eosinophilia, and prevent airway hyper-responsiveness, mucus hypersecretion and Th2 cytokine production $[7,8]$.

@ERSpublications

Hypereosinophilia (blood eosinophil count $>1500$ per $\mathrm{mm}^{3}$ ) in asthma patients may hide some rare myeloid or lymphoid neoplasms. Their identification has prognostic and therapeutic relevance because of the potential for targeted therapy. http://bit.ly/2JCoVIA

Cite this article as: Tabèze L, Marchand-Adam S, Borie R, et al. Severe asthma with blood hypereosinophilia associated with JAK2 V617F mutation: a case series. Eur Respir J 2019; 53: 1802248 [https://doi.org/10.1183/13993003.02248-2018]. 
TABLE 1 Clinical characteristics of three patients with asthma and hypereosinophilia

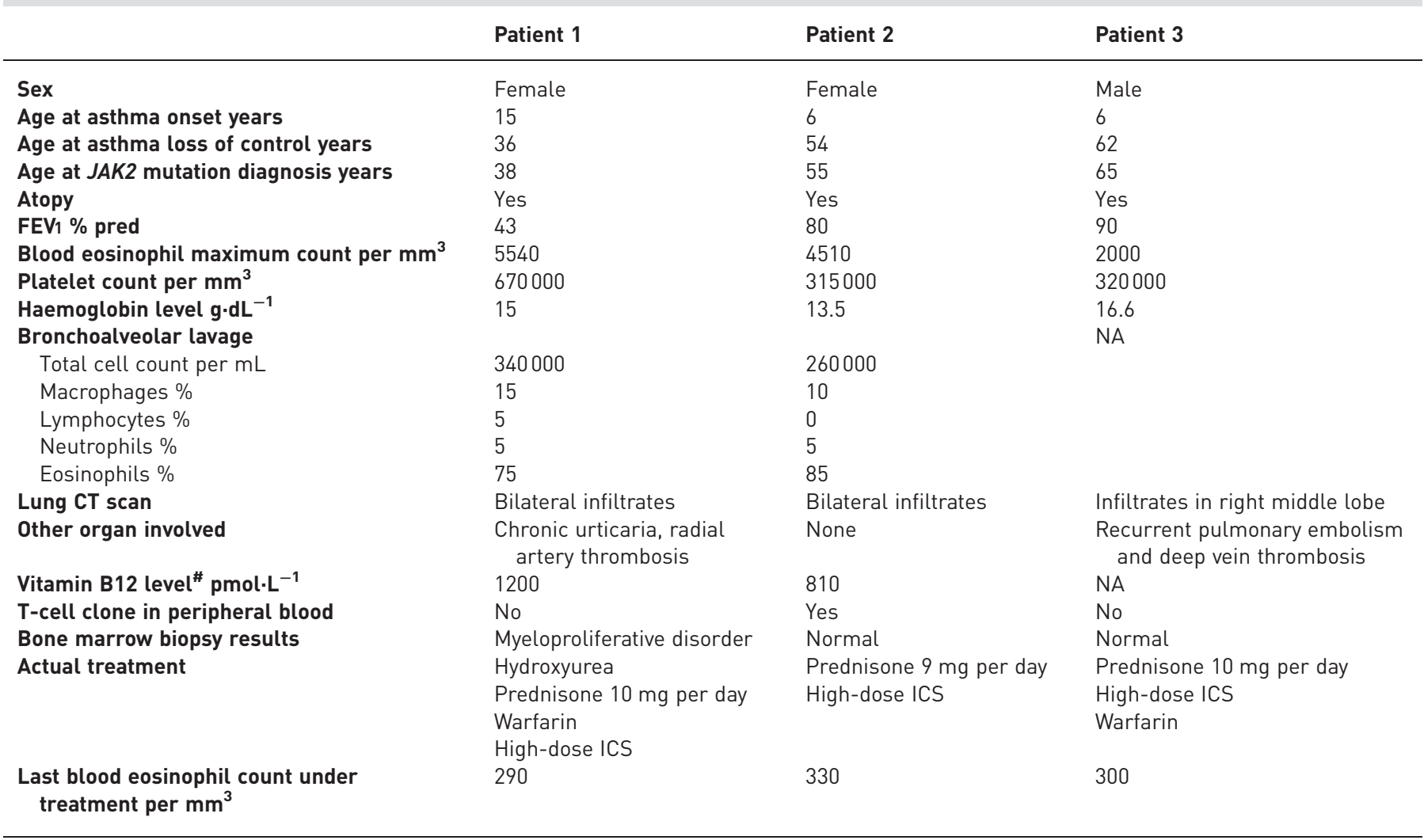

FEV1: forced expiratory volume in $1 \mathrm{~s}$; CT: computed tomography; ICS: inhaled corticosteroid therapy. ${ }^{\#}$ : normal values $139-651$ pmol.L ${ }^{-1}$.

The JAK2 mutation p.Val617Phe (V617F) induces a constitutively active protein and leads to myeloproliferation. The JAK2 V617F point mutation is found in $27 \%$ of cases of chronic myeloproliferative disorder [9], mainly polycythemia vera (PV) cancer and essential thrombocythemia (ET). HE may be observed in some cases of PV and ET, with higher values observed in JAK2 V617F-positive than -negative cases $[9,10]$, with the increase in granulocyte count depending on allele burden. JAK2 mutation is also detected in $1.5 \%$ to $4 \%$ of patients with $\mathrm{HE}$ of unknown significance, and is associated with a poor prognosis $[9,10]$.

All patients in our series had childhood asthma, but loss of control appeared later, simultaneously with HE and JAK2 mutation diagnosis. We cannot exclude an incidental finding, especially in patient 3 , who currently has no features of haematological neoplasm and may have a low allele burden, which was not assessed. However, given the known role of JAK2 in eosinophil activation and asthma pathophysiology [7, 8], we consider that JAK2 mutation, at least in our patients, may contribute to increased blood eosinophilia count and therefore asthma severity.

In our centre, we did not find any other haematological neoplasm associated with HE, probably because of recruitment bias, since haematological disorders with $\mathrm{HE}$ are rarely associated with localised disease such as asthma or eosinophilic pulmonary infiltrates at disease onset. For example, in a small series of 18 asthma patients with $\mathrm{HE}$, only one had a clonal T-cell receptor-gamma rearrangement [11]. One case of severe asthma in a child revealing a lymphoid variant of HE syndrome has been reported [12].

Our case series, with the limitation of bias due to its retrospective design, emphasises that HE in severe asthma patients may hide some rare haematological neoplasms, with lung manifestations as a single organ involvement. Blood eosinophilia $>2000$ per $\mathrm{mm}^{3}$, pulmonary infiltrates, persistence of high blood eosinophil counts under oral steroids, history of venous or arterial thrombosis, or increased platelet count should signal a possible haematological neoplasm and trigger a thorough evaluation.

This evaluation seems important because in patients with severe asthma, new biological therapies targeting the Th2 response specifically target disease with increased blood eosinophil count [13]. Mepolizumab has demonstrated clinical benefit in conditions with high eosinophil count other than asthma, such as HE 
syndrome [14] and eosinophilic granulomatosis with polyangiitis [15]. Despite the rarity of those molecular aberrations, their identification has prognostic and therapeutic relevance because of the potential for targeted therapy with JAK inhibitors [5, 7]. In patients with severe asthma and recurrent HE, screening for lympho- or myeloproliferative disorders (at least blood smear examination, tryptase and vitamin B12 dosage [3]) should be part of the evaluation.

Laure Tabèze ${ }^{1}$, Sylvain Marchand-Adam ${ }^{2,3}$, Raphaël Borie $\odot^{1}$, Aurélien Justet ${ }^{1}$, Clairelyne Dupin ${ }^{1}$, MarieChristine Dombret ${ }^{1}$, Bruno Crestani ${ }^{1}$ and Camille Taillé ${ }^{1}$

${ }^{1}$ Service de Pneumologie et Centre de Référence Constitutif des Maladies Pulmonaires Rares, Hôpital Bichat, Assistance Publique-Hôpitaux de Paris, Département Hospitalo-Universitaire FIRE, Université Paris Diderot, INSERM UMR 1152, LabEx Inflamex, Paris, France. ${ }^{2}$ Département de Pneumologie et Explorations fonctionnelles respiratoires, Centre Hopitalo Universitaire de Tours, Tours, France. ${ }^{3}$ INSERM U-1100, Faculté de médecine, Université François Rabelais, Tours, France.

Correspondence: Camille Taillé, Service de pneumologie, Hôpital Bichat, 46 rue Henri Huchard, 75018, Paris, France. E-mail: camille.taille@aphp.fr

Received: Nov 292019 | Accepted after revision: March 032019

Conflict of interest: L. Tabèze has nothing to disclose. S. Marchand-Adam has nothing to disclose. R. Borie reports grants and personal fees from Boehringer Ingelheim and Roche, outside the submitted work. A. Justet has nothing to disclose. C. Dupin has nothing to disclose. M-C. Dombret has nothing to disclose. B. Crestani reports research and travel grants and personal fees for lecturing from Boehringer Ingelheim and Roche, travel grants from Apellis, personal fees for travel and lecturing from AstraZeneca and Sanofi, grants from Medimmune, outside the submitted work. C. Taillé reports personal fees from and acting as study investigator for AstraZeneca, Boehringer, Novartis and ALK, personal fees from Chiesi and Teva, grants and personal fees from and acting as study investigator for GSK, acting as study investigator for Sanofi, outside the submitted work.

\section{References}

1 Price DB, Rigazio A, Campbell JD, et al. Blood eosinophil count and prospective annual asthma disease burden: a UK cohort study. Lancet Respir Med 2015; 3: 849-858.

2 Weissler JC. Eosinophilic lung disease. Am J Med Sci 2017; 354: 339-349.

3 Groh M, Pagnoux C, Baldini C, et al. Eosinophilic granulomatosis with polyangiitis (Churg-Strauss) (EGPA) Consensus Task Force recommendations for evaluation and management. Eur J Intern Med 2015; 26: 545-553.

4 Watanabe S, Arai Ki. Roles of the JAKSTAT system in signal transduction via cytokine receptors. Curr Opin Genet Dev 1996; 6: 587-596.

5 Monahan J, Siegel N, Keith R, et al. Attenuation of IL-5-mediated signal transduction, eosinophil survival, and inflammatory mediator release by a soluble human IL-5 receptor. J Immunol 1997; 159: 4024-4034.

6 Tripathi P, Hong X, Caruso D, et al. Genetic determinants in the development of sensitization to environmental allergens in early childhood. Immun Inflamm Dis 2014; 2: 193-204.

$7 \quad$ Kudlacz E, Conklyn M, Andresen C, et al. The JAK-3 inhibitor CP-690550 is a potent anti-inflammatory agent in a murine model of pulmonary eosinophilia. Eur J Pharmacol 2008; 582: 154-161.

8 Ashino S, Takeda K, Li H, et al. Janus kinase $1 / 3$ signaling pathways are key initiators of $\mathrm{TH} 2$ differentiation and lung allergic responses. J Allergy Clin Immunol 2014; 133: 1162-1174.e4.

9 Jones AV, Kreil S, Zoi K, et al. Widespread occurrence of the JAK2 V617F mutation in chronic myeloproliferative disorders. Blood 2005; 106: 2162-2168.

10 Schwaab J, Umbach R, Metzgeroth G, et al. KIT D816V and JAK2 V617F mutations are seen recurrently in hypereosinophilia of unknown significance. Am J Hematol 2015; 90: 774-777.

11 Freymond N, Kahn J-E, Legrand F, et al. Clonal expansion of T cells in patients with eosinophilic lung disease. Allergy 2011; 66: 1506-1508.

12 Leu T, Rauthe S, Wirth C, et al. Ein T-Zell assoziiertes Hypereosinophilie-Syndrom (L-HES) als Differenzialdiagnose eines Asthma bronchiale [The lymphoid variant of HES (L-HES) as differential diagnose of severe asthma in childhood]. Klin Padiatr 2016; 228: 319-324.

13 Israel E, Reddel HK. Severe and difficult-to-treat asthma in adults. N Engl J Med 2017; 377: 965-976.

14 Kuang FL, Fay MP, Ware J, et al. Long-term clinical outcomes of high-dose mepolizumab treatment for hypereosinophilic syndrome. J Allergy Clin Immunol Pract 2018; 6: 1518-1527.

15 Wechsler ME, Akuthota P, Jayne D, et al. Mepolizumab or placebo for eosinophilic granulomatosis with polyangiitis. N Engl J Med 2017; 376: 1921-1932. 